\title{
Sosiho-tang ameliorates cachexia-related symptoms in mice bearing colon 26 adenocarcinoma by reducing systemic inflammation and muscle loss
}

\author{
AEYUNG KIM*, MINJU IM* and JIN YEUL MA \\ Korean Medicine (KM) Application Center, Korea Institute of Oriental Medicine (KIOM), \\ Dong-gu, Daegu 701-300, Republic of Korea
}

Received September 11, 2015; Accepted October 20, 2015

DOI: $10.3892 /$ or.2015.4527

\begin{abstract}
Cachexia accompanied by muscle wasting is a key determinant of poor prognosis in cancer patients and cancer-related death. Previous studies have demonstrated that inflammatory cytokines such as interleukin-6 (IL-6), tumor necrosis factor- $\alpha$ (TNF- $\alpha)$, IL-1 and interferon- $\gamma$ (IFN- $\gamma)$ secreted from host cells and tumor cells participate in skeletal muscle wasting followed by severe loss of body weight. Therefore, blockade of the inflammatory response is thought to be a logical target for pharmacological and nutritional interventions to preserve skeletal muscle mass under cachectic conditions. Sosiho-tang (SO; Xiaocharihu-tang in Chinese and Sho-saiko-to in Japanese) is an Oriental herbal medicine that has been used to treat chronic hepatic diseases and to control fever. In recent studies, SO inhibited the production of inflammatory cytokines in lipopolysaccharide (LPS)-stimulated macrophages, prevented thrombus formation and suppressed cancer progression. However, the anti-cachectic activity of SO in tumor-bearing mice has not yet been examined. In the present study, we characterized the effect of SO administration on cancer-induced cachexia in CT-26-bearing mice, and elucidated the anti-cachectic mechanisms. Daily oral administration of SO at doses of 50 and $100 \mathrm{mg} / \mathrm{kg}$ to CT-26-bearing mice significantly retarded tumor growth and prevented the loss of final body weight, carcass weight, heart weight, gastrocnemius muscle, and epididymal fat, compared with saline-treated control mice. In addition, serum IL-6 levels elevated by cancer were decreased by SO administration. In the J774A.1 macrophage cell line, SO efficiently suppressed LPS-mediated increases
\end{abstract}

Correspondence to: Dr Jin Yeul Ma, Korean Medicine (KM) Application Center, Korea Institute of Oriental Medicine (KIOM), 70 Chumdan-ro, Dong-gu, Daegu 701-300, Republic of Korea E-mail: jyma@kiom.re.kr

*Contributed equally

Key words: Oriental herbal medicine, Sosiho-tang, cancer cachexia, weight loss, inflammation in inducible nitric oxide synthase (iNOS) expression, nitric oxide (NO), and procachectic inflammatory cytokine production through inhibition of nuclear factor- $\kappa \mathrm{B}(\mathrm{NF}-\kappa \mathrm{B})$ and $\mathrm{p} 38$ activation. In addition, $\mathrm{SO}$ attenuated muscle atrophy caused by cancer cells by affecting myoblast proliferation and differentiation, and $\mathrm{C} 2 \mathrm{C} 12$ myotube wasting. Taken together, these results suggest that $\mathrm{SO}$ is a safe and useful anti-cachectic therapy for cancer patients with severe weight loss.

\section{Introduction}

Cancer cachexia is a severe, debilitating and life-threatening syndrome, characterized by a marked loss of skeletal muscle and adipose tissue and appetite, a reduction in physical functions, and profound involuntary weight loss despite adequate nutritional intake (1-3). Weight loss is more frequently experienced by $80-90 \%$ of pancreatic and gastrointestinal cancer patients than by sarcoma and breast cancer patients, and severe cachexia with $>10 \%$ weight loss is closely correlated with high mortality, impaired quality of life and poor response to chemotherapy or radiotherapy (4). In addition, cachectic patients are more prone to chemotherapy-related toxic side effects. Therefore, it is important to control the cachectic state in cancer patients, since cachexia is the main cause of cancer-related death in approximately $25-30 \%$ of patients.

Although the mechanisms of cancer cachexia are not completely known, recent studies have indicated that persistent production of proinflammatory cytokines and catabolic factors secreted from both tumor cells and host cells, such as macrophages, plays an essential role in the induction and progression of cancer cachexia $(5,6)$. Among procachectic cytokines, interleukin-6 (IL-6) is considered a key mediator in the pathogenesis of cancer cachexia. In previous studies, it has been reported that IL-6-secreting cells induce wasting of both muscle and fat, and IL-6 is responsible for muscle wasting in cachectic mice with CT-26 or Yomoto uterine cancer (7). Treatment with monoclonal antibodies to IL-6, or to the IL-6 receptor, markedly suppresses the development of cachexia in tumor-bearing mice and in patients with IL-6-overexpressing lung cancer, suggesting that blocking IL-6 function might be an effective intervention for the management of cachectic patients $(8,9)$. 
Several clinical trials to treat cancer cachexia have been performed, involving drugs that stimulate appetite, reduce proinflammatory cytokine production and inhibit tumor-induced protein degradation. However, current medical treatments are limited due to low efficacy and high toxicity. Among them, medroxyprogesterone acetate, approved in Europe for treatment of cancer and acquired immune deficiency syndrome (AIDS)-related cachexia, is widely used in the treatment of hormone-related cancer as a supportive therapy $(10,11)$. However, this treatment also has unwanted side effects, including diabetes, osteoporosis, mood swings and thromboembolism (12). Therefore, it is essential to find novel agents capable of preventing cancer-induced cachexia with minimal adverse effects.

Sosiho-tang (SO, known as Xiaochaihu-tang in Chinese, and as Sho-saiko-to in Japanese) is an Oriental traditional herbal formula comprised of seven medicinal herbs, including Bupleurum root, Glycyrrhizae radix et rhizoma, Ginseng, radix, Pinellia tuber, Scutellaria root, Zingiberis rhizoma crudus and Zizyphi fructus. SO has long been used to treat chronic liver diseases such as hepatitis and liver cirrhosis in Korea, China and Japan (13). In addition, SO prevents inflammation and cancer progression (14-17), suggesting that it might also prevent cancer-induced cachexia through suppression of inflammatory responses caused by cancer.

In the present study, we examined the effects of oral administration of SO on the severity of key parameters of cachexia in CT-26-bearing mice. In addition, we also investigated the efficacy of SO on the production of proinflammatory cytokines and muscle wasting, and further elucidated the in vitro mechanism of its anti-cachectic activity using murine cell lines, including the CT-26 colon carcinoma, the J774A.1 macrophage, and the $\mathrm{C} 2 \mathrm{C} 12$ myoblast cell lines.

\section{Materials and methods}

Cells. Murine colon carcinoma CT-26 cells and murine myoblast $\mathrm{C} 2 \mathrm{C} 12$ cells were purchased from the American Type Culture Collection (Manassas, VA, USA). Murine macrophage-like J774A.1 cells [Korean Cell Line Bank (KCLB): no. 40067] were obtained from the KCLB (Seoul, Korea). The cells were maintained in Dulbecco's modified Eagle's medium (DMEM) or Roswell Park Memorial Institute (RPMI)-1640 medium (Lonza, Walkersville, MD, USA) supplemented with 10\% (v/v) heat-inactivated fetal bovine serum (FBS; Cellgro, Manassas, VA, USA) and penicillin $(100 \mathrm{U} / \mathrm{ml}) /$ streptomycin $(100 \mu \mathrm{g} / \mathrm{ml})$ (Cellgro) in a humidified $5 \% \mathrm{CO}_{2}$ incubator at $37^{\circ} \mathrm{C}$. To induce myogenic differentiation, the $\mathrm{C} 2 \mathrm{C} 12$ cells at a density of $70-80 \%$ were cultured in DMEM containing 5\% horse serum (HS; Gibco-BRL, Grand Island, NY, USA) for 5-7 days.

Animals. Six-week-old male BALB/c mice were purchased from Taconic Farms (Samtako Bio Korea, Osan, Korea) and housed under specific pathogen-free conditions under a 12-h light-dark cycle at $22 \pm 1^{\circ} \mathrm{C}$ and $55 \pm 5 \%$ humidity. All animal experiments were approved by the Animal Care and Use Committee of the Korea Institute of Oriental Medicine (KIOM, Daejeon, Korea) with reference numbers \#13-100,\#14-074 and \#15-011. Experiments were performed according to the guidelines of the Animal Care and Use Committee at KIOM.
Table I. Herbal composition of Sosiho-tang.

\begin{tabular}{lrl}
\hline Name of herb & $\begin{array}{c}\text { Amount } \\
(\mathrm{g})\end{array}$ & \multicolumn{1}{c}{$\begin{array}{c}\text { Location } \\
\text { of origin }\end{array}$} \\
\hline Bupleurum root & 12.00 & Yeongcheon, Korea \\
Glycyrrhizae radix et rhizoma & 2.00 & China \\
Ginseng radix & 4.00 & Geumsan, Korea \\
Pinellia tuber & 4.00 & Uiseong, Korea \\
Scutellaria root & 8.00 & Suncheon, Korea \\
Zingiberis rhizoma crudus & 1.49 & Yeongcheon, Korea \\
Zizyphi fructus & 2.00 & Yeongcheon, Korea \\
Total amount & 33.49 & \\
\hline
\end{tabular}

Antibodies and reagents. Antibodies against $\mathrm{p} 21$, cyclin-dependent kinase (CDK)2, cyclin D, p38, p-p38 (Thr180/Tyr182), $\mathrm{p}-\mathrm{I} \kappa \mathrm{B} \alpha$ (Ser32), p-IKK $\alpha / \beta$ (Ser176/180), signal transducer and activator of transcription (STAT)3, p-STAT3 (Tyr705), p65, p-p65 (Ser536), inducible nitric oxide synthase (iNOS), and TBP were purchased from Cell Signaling Technology (Danvers, MA, USA). Anti- $\alpha$-tubulin antibody was obtained from Santa Cruz Biotechnology (Santa Cruz, CA, USA). Antibody against myosin heavy chain was purchased from R\&D Systems (Minneapolis, MN, USA). Horseradish peroxidase (HRP)-conjugated anti-mouse and anti-rabbit antibodies were purchased from Cell Signaling Technology. Recombinant murine tumor necrosis factor- $\alpha$ (rMu TNF- $\alpha$ ) was purchased from Promokine (Heidelberg, Germany).

Preparation of SO. The composition of herbal extract SO is listed in Table I. All herbs were obtained from Yeongcheon Herbal Market (Yeongcheon, Korea), validated by Professor Ki Hwan Bae (Chungnam National University, Daejeon, Korea), and stored in the herbal bank at KIOM prior to use. A total of $1,674.5 \mathrm{~g}$ of chopped SO was heat-extracted in 16.745 liters of distilled water for $3 \mathrm{~h}$ at $115^{\circ} \mathrm{C}$ using a Cosmos-600 Extractor (Gyungseo, Incheon, Korea). The decoction was filtered through standard testing sieves $(150 \mu \mathrm{m}$; Retsch, Haan, Germany), lyophilized, and stored in desiccators at $4^{\circ} \mathrm{C}$. The final amount of lyophilized SO powder was $416.783 \mathrm{~g}$, and the yield was $24.89 \%$. For in vitro experiments, the SO powder was dissolved in $10 \%$ dimethyl sulfoxide to $50 \mathrm{mg} / \mathrm{ml}$, filtered through a $0.22-\mu \mathrm{m}$ disk filter, and then stored at $-20^{\circ} \mathrm{C}$ prior to use.

Experimental design of cancer cachexia. The CT-26 cells were subcutaneously inoculated into the abdominal region of 7 -week-old male BALB/c mice ( $5 \times 10^{6}$ cells/mouse). To confirm the induction of cancer-mediated cachexia, the body weights and tumor volumes of the mice were measured once every 2 days throughout the experiment. Significant weight losses in the tumor-bearing mice were observed between 8-10 days after the tumor inoculations, and the mice were fed with saline or SO at doses of 50 and $100 \mathrm{mg} / \mathrm{kg}$ from day 10 to day 27 after tumor inoculation. The administered dose was calculated based on the amount used in human adults $(33.49 \mathrm{~g} / 60 \mathrm{~kg}$ of body weight/day) and the yield of powdered extract (24.89\%). The age-matched healthy control mice having no tumors were 
treated with saline. During the experiments, food intake was calculated as the mean value of five mice per cage. At the time of sacrifice, mice were euthanized by intraperitoneal injection with a 2:1 mixture of zoletil (Virbac, Magny-en-Vexin, France) and rompun (Bayer, Seoul, Korea), and then the tumor, epididymal fat, gastrocnemius muscle, and heart were dissected and weighed. In addition, serum samples were obtained for measuring levels of IL-6, tumor necrosis factor (TNF)- $\alpha$, and IL-1 $\beta$. After exsanguination, the remaining viscera were removed and the carcass weight was measured.

Preparation of CT-26 conditioned medium (CM). The CT-26 cells were plated into $100-\mathrm{mm}$ culture dishes at a density of $5 \times 10^{4}$ cells $/ \mathrm{cm}^{2}$ and treated with or without SO for $24 \mathrm{~h}$ under complete medium conditions. After washing three times with phosphate-buffered saline (PBS), the cells were additionally washed twice with serum-free medium, and then incubated for another $24 \mathrm{~h}$ in serum-free DMEM. The resulting CM was centrifuged to remove debris, filtered using a $0.22-\mu \mathrm{m}$ disk filter, and then stored in a freezer. The CM was diluted at a 1:3 or 1:5 ratio with either DMEM containing 10\% FBS and antibiotics [growth medium (GM)] for myoblast treatment or DMEM containing 5\% HS and antibiotics [differentiation medium (DM)] for the myotube treatment. Prior to dilution, an appropriate quantity of FBS, HS, or antibiotics for compensation were added to the CM.

Myoblast proliferation assay. To examine the C2C12 myoblast proliferation, cells were plated into a 96 -well culture plate at a density of $1 \times 10^{3}$ cells/well, and then treated with SO-treated or -untreated $\mathrm{CM}$ at $37^{\circ} \mathrm{C}$ for $48 \mathrm{~h}$. At the indicated time points, cell proliferation was determined using the Cell Counting Kit-8 (Dojindo Laboratories, Kumamoto, Japan) according to the manufacturer's protocol.

Determination of NO production. The cells were pretreated with the indicated concentrations of SO for $1 \mathrm{~h}$ and then stimulated with LPS for $24 \mathrm{~h}$. The collected culture supernatant was mixed with an equal volume of Griess reagent (1\% sulfanilamide, $0.1 \%$ naphthylethylenediamine dihydrochloride and $2.5 \%$ phosphoric acid), and incubated at room temperature for $5 \mathrm{~min}$, and then the absorbance was measured at $570 \mathrm{~nm}$.

Reverse transcription-polymerase chain reaction (RT-PCR). The total RNA was isolated using an RNA extraction solution (BioAssay Co., Daejeon, Korea) according to the manufacturer's instructions, and the RNA concentrations were quantitated using a NanoDrop ND-1000 spectrophotometer (NanoDrop Technologies, Wilmington, DE, USA). The RNA (3 $\mu \mathrm{g})$ was reverse transcribed using a $1^{\text {st }}$ Strand cDNA Synthesis kit (BioAssay Co.) and then the cDNA samples were analyzed by semiquantitative PCR using specific primers (Table II). PCR products were visualized by electrophoresis using agarose gels and staining with GreenLight ${ }^{\mathrm{TM}}$ (BioAssay Co.), and band intensities were analyzed using ImageJ software (National Institutes of Health, Bethesda, MD, USA).

Western blot analysis. For whole cell lysates, cells were harvested, washed and lysed in M-PER Mammalian Protein Extraction Reagent (Thermo Scientific, Rockford, IL, USA).
Table II. Primers used for PCR.

\begin{tabular}{lc}
\hline Target gene & Sequences \\
\hline iNOS & F: 5'-CCTCCTCCACCCTACCAAGT-3' \\
& R: 5'-CACCCAAAGTGCTTCAGTCA-3' \\
IL-6 & F: 5'-CATGTTCTCTGGGAAATCGTGG-3' \\
& R: 5'-AACGCACTAGGTTTGCCGAGTA-3' \\
TNF- $\alpha$ & F: 5'-CATGTTCTCTGGGAAATCGTGG-3' \\
& R: 5'-AACGCACTAGGTTTGCCGAGTA-3' \\
IL-1 $\alpha$ & F: 5'-GGTTAAATGACCTGCAACAGGA-3' \\
& R: 5'-TCTTTGGTGGCAATAAACAGC-3' \\
GAPDH & F: 5'-TCATGACCACAGTCCATGCC-3' \\
& R: 5'-TCCACCACCCTGTTGCTGTA-3'
\end{tabular}

F, forward; R, reverse.

The cytosolic and nuclear fractions were obtained using NE-PER Nuclear and Cytoplasmic Extraction Reagent (Thermo Scientific). The lysates were separated using sodium dodecyl sulfate polyacrylamide gel electrophoresis (SDS-PAGE), followed by transfer onto a polyvinylidene fluoride (PVDF) membrane (Bio-Rad, Hercules, CA, USA), then blocked with $3 \%$ bovine serum albumin (BSA) in Tris-buffered saline containing $0.05 \%$ Tween-20 (TBST) and immunoblotted using specific antibodies at $4^{\circ} \mathrm{C}$ overnight. After washing with TBST, the membranes were reacted with secondary antibodies conjugated with HRP for $1 \mathrm{~h}$ at room temperature, and the target proteins were visualized using the SuperSignal WestFemto Maximum Sensitivity Substrate (Pierce, Rockford, IL, USA) and the ImageQuant LAS 4000 Mini (GE Healthcare, Piscataway, NJ, USA). The relative band intensities were measured using ImageJ software.

Enzyme-linked immunosorbent assay (ELISA). The levels of murine IL-6, TNF- $\alpha$ and IL- $1 \beta$ in the culture supernatants and sera were determined using an ELISA antibody kit (eBioscience, San Jose, CA, USA) according to the manufacturer's instructions.

Statistical analysis. Data are presented as means \pm standard deviation (SD). Differences between groups were analyzed by the Student's t-test using the SigmaPlot software (ver 8.0; SPSS Inc., Chicago, IL, USA). $\mathrm{P}<0.05$ was considered to indicate a statistically significant difference.

\section{Results}

Oral administration of SO alleviates cachexia symptoms in CT-26 tumor-bearing mice and suppresses tumor growth. To examine whether SO is an effective treatment for cancer-induced cachexia, mice were administered SO from 10 days after CT-26 cell inoculation, when mice showed reductions in body weight and food intake by $\sim 10-12 \%$ compared to the normal mice (Fig. 1). Normal mice exhibited gradually increased body weight during the experimental period by $21.6 \%$, while control mice had increased body weight by $4.3 \%$ (Fig. 2A). SO administration at doses of 50 and $100 \mathrm{mg} / \mathrm{kg}$ significantly 


\begin{tabular}{|c|c|c|}
\hline Day 0 & Day 10 & Day 27 \\
\hline $\begin{array}{l}\text { CT-26 s.c injection } \\
\left(5 \times 10^{6} \text { cells } / \text { mouse }\right)\end{array}$ & $\begin{array}{l}\text { Oral administration } \\
\text { with SO daily }\end{array}$ & $\begin{array}{c}\text { Sacrifice mice } \\
\text { analysis }\end{array}$ \\
\hline $\begin{array}{l}\text { Balb/c mice (male) } \\
\text { No tumor }(\mathrm{n}=5) \\
\text { Tumor }(\mathrm{n}=15)\end{array}$ & 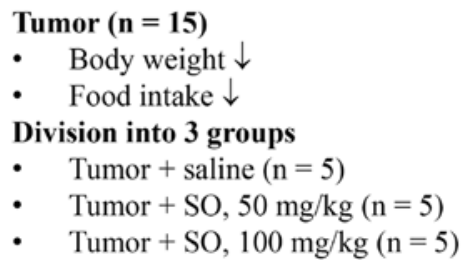 & $\begin{array}{l}\text { Body weight } \\
\text { Food intake } \\
\text { Muscle weight } \\
\text { Fat weight } \\
\text { Cytokine level }\end{array}$ \\
\hline
\end{tabular}

Figure 1. Experimental protocol for the treatment of cancer-induced cachexia. Balb/c mice $(\mathrm{n}=15)$ were subcutaneously injected with $5 \times 10^{6} \mathrm{CT}-26$ cells in the abdominal region. On day 10 after tumor injection, when cachexia-related symptoms including decrease in body weight and food intake were observed, the mice were randomly divided into three groups ( $\mathrm{n}=5$ per group) and administered daily with 50 and $100 \mathrm{mg} / \mathrm{kg}$ Sosiho-Tang (SO) and saline (control) in a volume of $100 \mu \mathrm{l}$. Normal mice having no tumors $(\mathrm{n}=5)$ were administered daily with the same volume of saline during the experiment. On day 27 after the tumor injection, mice were sacrificed and analyzed for cachectic parameters.

A

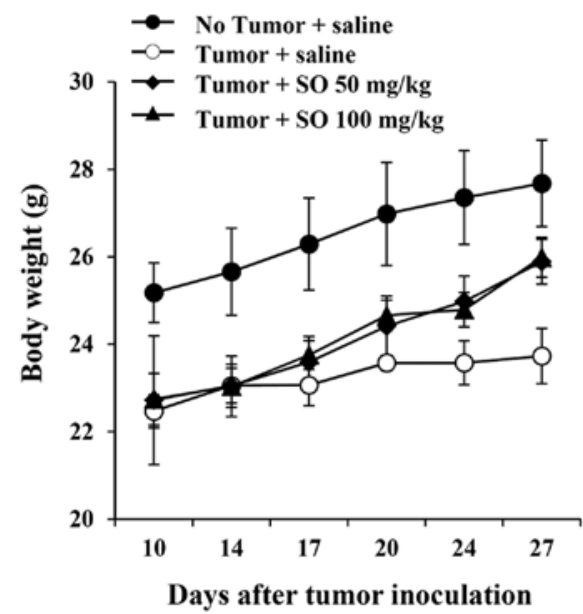

C
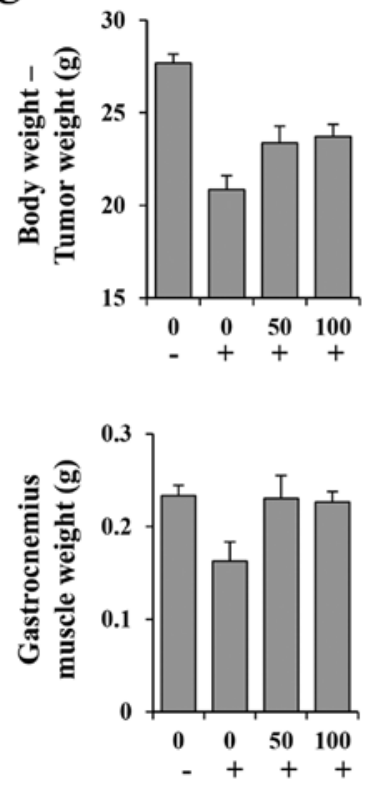

B
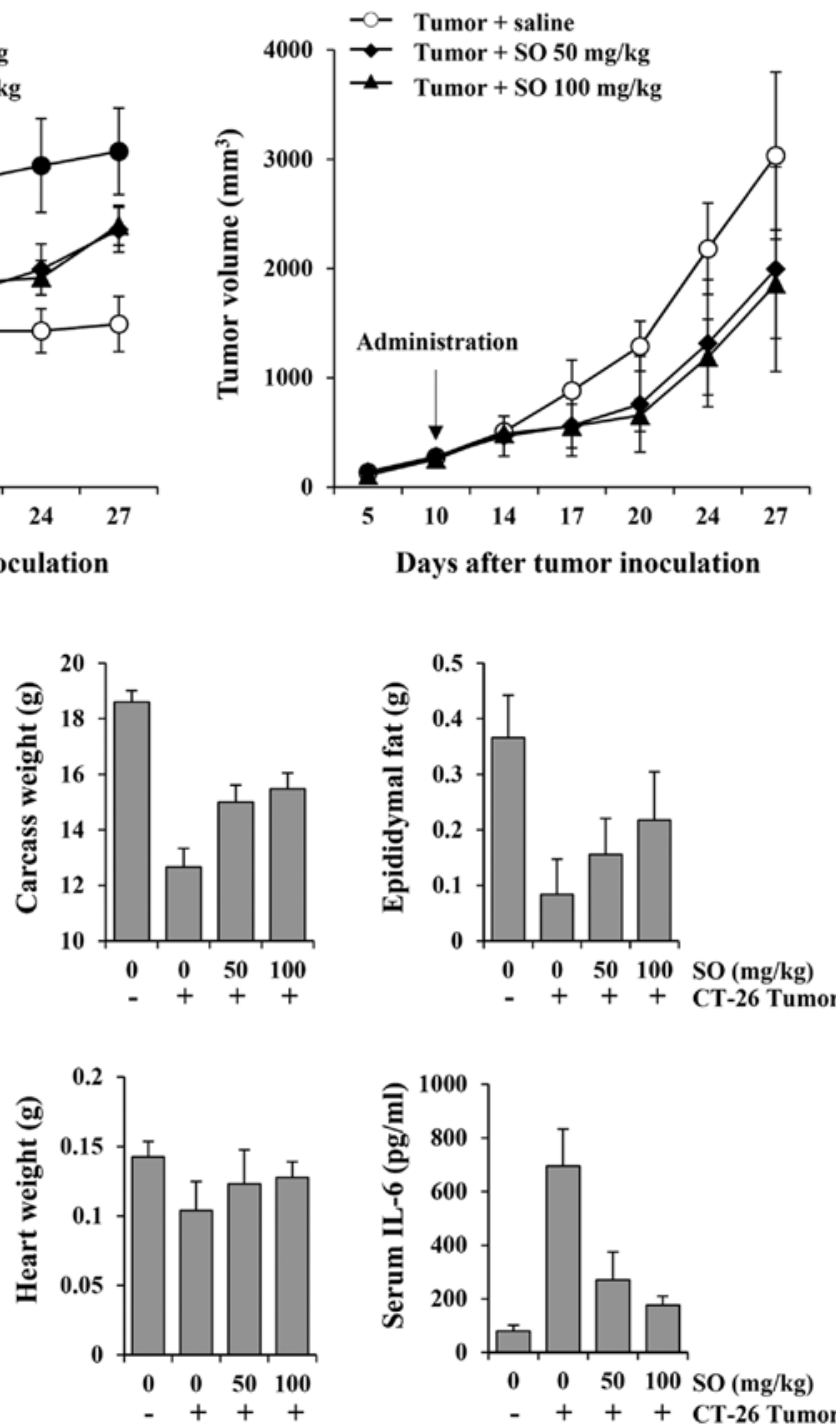

Figure 2. Effects of SO administration on body weight, tumor growth, nutritional parameters and serum interleukin (IL)-6 levels in CT-26 tumor-bearing mice. On day 10 after tumor inoculation, the mice were administered daily with SO at doses of 50 and $100 \mathrm{mg} / \mathrm{kg}$, or saline for 17 consecutive days. The healthy control mice with no tumors were also treated daily with saline. The body weight, tumor size, and food intake were measured on days $14,17,20$, 24 and 27 (A and B). After mice were sacrificed, the carcass, epididymal fat, gastrocnemius muscle, and heart were weighed, and serum IL-6 levels were measured using an enzyme-linked immunosorbent assay (ELISA) (C). Animal experiments were performed three times. Representative results are shown. 
A

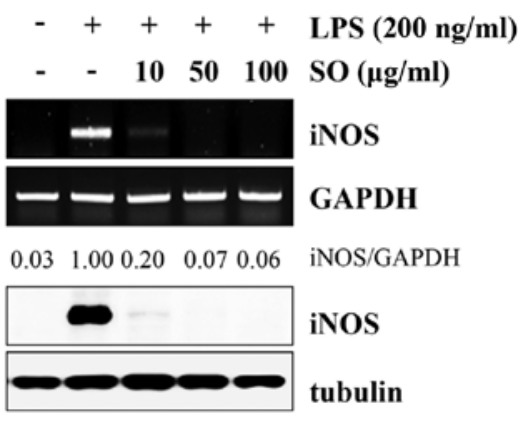

$\begin{array}{lllll}0.02 & 1.00 & 0.04 & 0.01 & 0.01 \quad \mathrm{iNOS} / \text { tubulin }\end{array}$
B
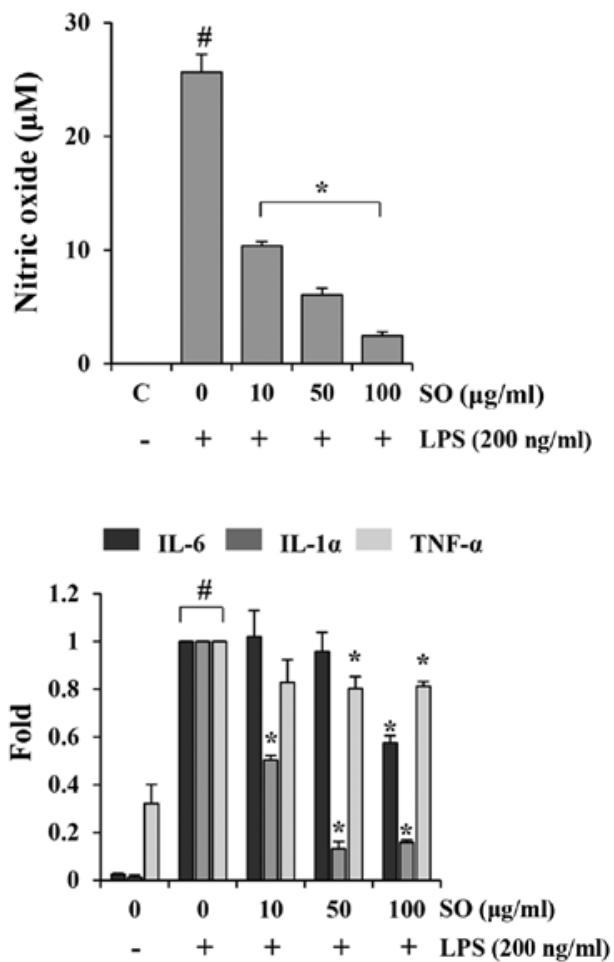

D

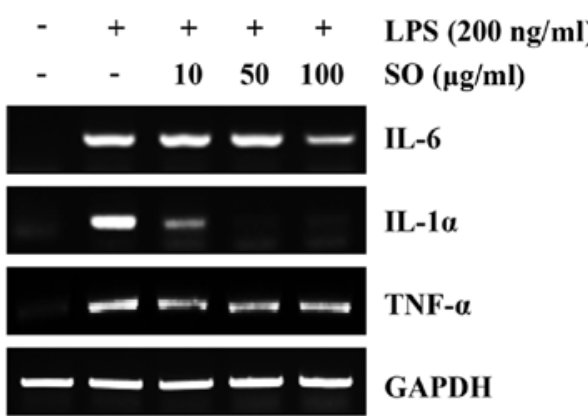

LPS (200 ng/ml)

\section{C}
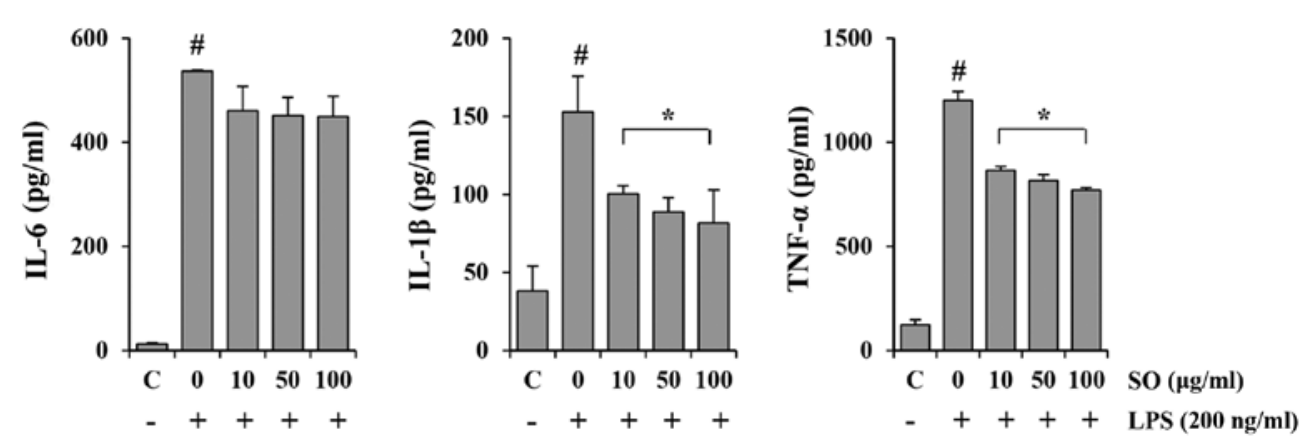

Figure 3. The inhibitory effects of SO on lipopolysaccharide (LPS)-induced nitric oxide (NO) production and inflammatory cytokine production in murine macrophage J774A.1 cells. (A) The J774A.1 cells were pretreated with indicated concentrations of SO for $1 \mathrm{~h}$, and then stimulated with $200 \mathrm{ng} / \mathrm{ml} \mathrm{LPS}$ for $24 \mathrm{~h}$. The inducible nitric oxide synthase (iNOS) mRNA and protein levels were examined by RT-PCR and western blotting, respectively. The band intensities relative to LPS-stimulated cells were calculated using the ImageJ software. The levels of glyceraldehyde 3-phosphate dehydrogenase (GAPDH) and tubulin were measured for normalization. Data are representative of three independent experiments. (B) The culture supernatants were collected and analyzed for NO production. The control cells were treated with vehicle alone. The data are representative of three independent experiments performed in triplicate and are expressed as means $\pm \mathrm{SD}$. ${ }^{\prime} \mathrm{P}<0.05$ vs. untreated control, ${ }^{*} \mathrm{P}<0.05$ vs. SO-untreated control cells. (C) The J774A.1 cells pretreated with SO for $1 \mathrm{~h}$ were stimulated with $200 \mathrm{ng} / \mathrm{ml}$ LPS for $6 \mathrm{~h}$. The mRNA levels for interleukin (IL)-6, IL-1 $\alpha$, and tumor necrosis factor (TNF)- $\alpha$ were analyzed by RT-PCR, and relative fold increases were calculated after normalization with GAPDH using ImageJ software. The data show means \pm SD of two independent experiments.

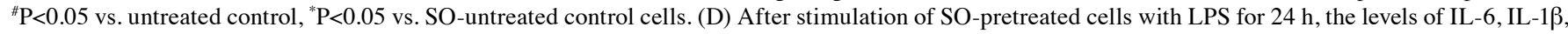
and TNF- $\alpha$ in the culture supernatants were quantitated by ELISA. Data are representative of three independent experiments performed in triplicate and are expressed as means \pm SD. ${ }^{~} \mathrm{P}<0.05$ vs. untreated control, ${ }^{*} \mathrm{P}<0.05$ vs. SO-untreated control cells.

increased body weights, by 13.1 and $13.7 \%$, respectively, showing recovery of body weights to $\sim 93.5$ and $93.9 \%$, respectively, of the normal mice. In previous studies, it has been reported that $\mathrm{SO}$ inhibits cell proliferation via cell cycle arrest at $\mathrm{G}_{0} / \mathrm{G}_{1}$, and induces apoptosis of various types of cancer cells, including hepatocellular carcinoma, Lewis lung carcinoma, cholangiocarcinoma, ovarian cell carcinoma and renal cell carcinoma $(15,16)$. In the present study, SO administration at 50 and $100 \mathrm{mg} / \mathrm{kg}$ significantly retarded CT-26 tumor growth, by 34.29 and $38.81 \%$, respectively, compared with the control mice on day 27. Control mice had a mean tumor weight of $2.88 \pm 0.89 \mathrm{~g}$, while mice treated with 50 and $100 \mathrm{mg} / \mathrm{kg}$ of SO had mean tumor weights of $2.52 \pm 0.61$ and $2.26 \pm 0.19 \mathrm{~g}$, reflecting 12.56 and $21.37 \%$ reductions, respectively (Fig. 2B). The mean values of food intake/mouse/day during the experiment for normal, control, and 50- and $100-\mathrm{mg} / \mathrm{kg} \mathrm{SO}$-treated mice were $3.56 \pm 0.11,3.13 \pm 0.24,3.32 \pm 0.16$ and $3.37 \pm 0.18 \mathrm{~g}$, respectively, indicating that $\mathrm{SO}$ aids in improving appetite (data not shown). In addition, administration of SO significantly prevented the loss of final body weight, carcass weight, heart 
A

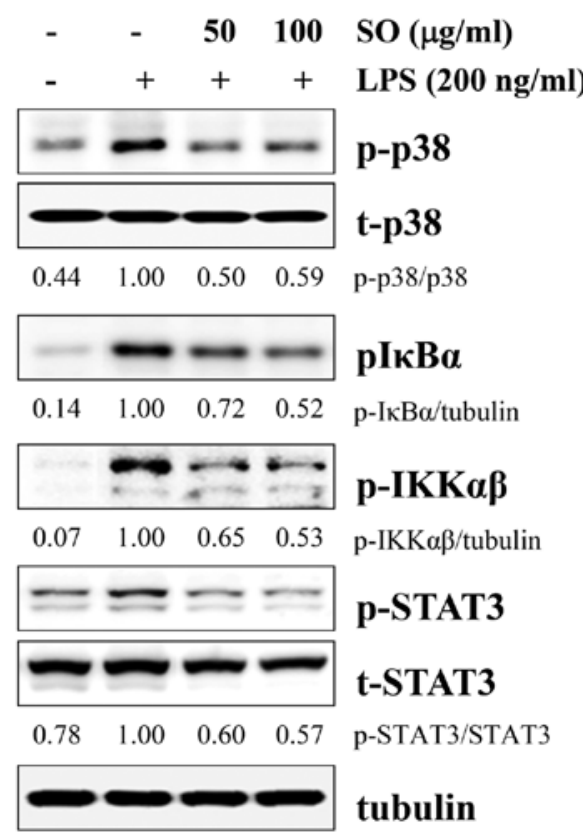

B
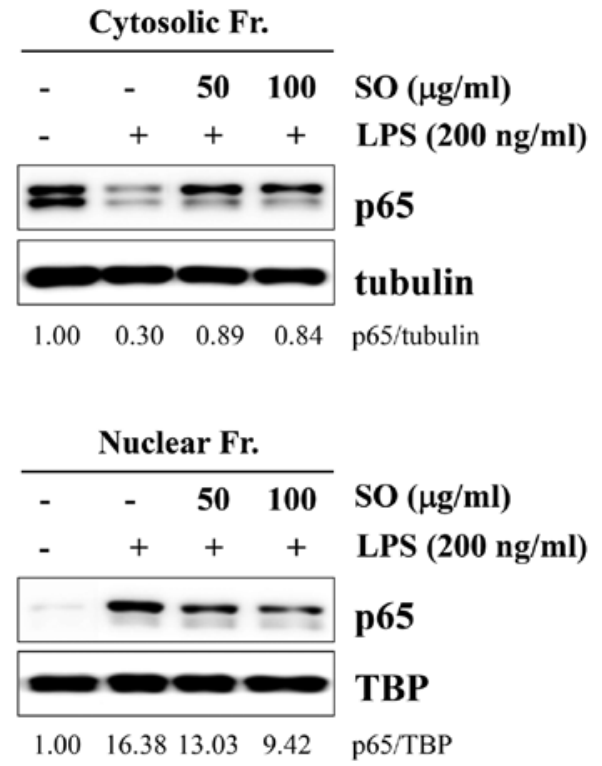

Figure 4. Effect of SO on LPS-induced p38 and STAT3 phosphorylation and NF- $\kappa \mathrm{B}$ activation in J774A.1 cells. (A) Cells pretreated with SO (50 or $100 \mu \mathrm{g} / \mathrm{ml})$ for $12 \mathrm{~h}$ were stimulated with LPS $(200 \mathrm{ng} / \mathrm{ml})$ for $30 \mathrm{~min}$ and then subjected to western blotting. The band intensities relative to SO-untreated control cells were calculated after normalization to tubulin expression. (B) The LPS-induced nuclear translocation of the NF- $\kappa$ B p65 subunit was measured by western blotting. Tubulin and TBP were used as loading controls for cytosolic and nuclear fractions, respectively.

weight and wasting of epididymal adipose tissue and gastrocnemius muscle in the CT-26 tumor-bearing mice. Furthermore, serum IL-6 levels were markedly elevated in the tumor-bearing control mice compared to the non-tumor-bearing normal mice, and these values were significantly reduced by SO administration (Fig. 2C). The serum levels of TNF- $\alpha$ and IL-1 $\beta$ were below the detection limit in all groups (data not shown). These results indicate that $\mathrm{SO}$ reduces tumor burden and delays the process of CT-26 tumor-induced cachexia.

SO downregulates LPS-induced NO production, iNOS expression and inflammatory cytokine production in murine macrophage J774A.1 cells. Fig. 2C shows that the serum level of IL-6 was markedly decreased by SO administration in the CT-26-bearing mice compared to the control mice. In cancer patients, the tumor induces a chronic host inflammatory response, characterized by the production of cytokines such as IL- 6 , IL- $1 \beta$, IFN- $\gamma$ and TNF- $\alpha$. These cytokines are known to be involved in the induction of cancer-related muscle wasting through activation of iNOS expression and NO production (18-20). Therefore, we examined whether SO could suppress iNOS expression, NO generation and inflammatory cytokine production in the LPS-stimulated J774A.1 cells. SO almost completely blocked the LPS-induced increase in iNOS expression at both the mRNA and protein levels (Fig. 3A). Furthermore, SO at 10, 50 and $100 \mu \mathrm{g} / \mathrm{ml}$ inhibited the NO production by 59.62, 76.43 and $90.42 \%$, respectively, in a dose-dependent manner, compared with the untreated controls (Fig. 3B). The mRNA levels of IL-6, IL-1 $\alpha$ and TNF- $\alpha$, as well as the protein levels of IL-6, IL-1 $\beta$ and TNF- $\alpha$, were strongly increased by LPS stimulation, and were efficiently suppressed by the SO treatment (Fig. 3C and D).
Similar to observations in J774A.1 cells, SO suppressed the LPS-induced NO production, iNOS expression, and cytokine production in peritoneal macrophages (data not shown). SO at the concentrations used in the experiments did not affect the viability of J774A.1 cells or primary peritoneal macrophages, excluding the possibility of cytotoxic effects.

SO strongly blocks LPS-induced p38, NF- $\kappa B$, and STAT3 activation in J774A.1 cells. Because mitogen-activated protein kinase (MAPK), NF- $\mathrm{B}$, and STAT3 activations are closely related with proinflammatory cytokines, we examined whether these pathways were affected by SO treatment. After LPS stimulation, the levels of phosphorylated p38, IкB $\alpha, \operatorname{IKK} \alpha \beta$, and STAT3 were significantly decreased by SO treatment, in a dose-dependent manner (Fig. 4A). However, the levels of phosphorylated ERK and JNK were not affected (data not shown). Because NF- $\kappa \mathrm{B}$ activation requires nuclear translocation of p65, we measured p65 levels in the cytosolic and nuclear fractions. In control cells, the p65 subunit translocated from the cytosol to the nucleus by LPS stimulation, whereas SO treatment effectively prevented p65 nuclear translocation in a dose-dependent manner (Fig. 4B).

SO attenuates CT-26-mediated skeletal muscle atrophy in murine $C 2 C 12$ myotubes. In cancer cachexia, proinflammatory cytokines including IL- 6 , IL- $1 \beta$, IFN- $\gamma$ and TNF- $\alpha$ have been implicated in the progression of skeletal muscle wasting (21). In addition to these humoral factors, tumor-derived factors such as myostatin and proteolysis-inducing factor collectively promote skeletal muscle wasting (22-24). In previous studies, it has been reported that CT-26 CM inhibited C2C12 myoblast proliferation and differentiation and stimulated $\mathrm{C} 2 \mathrm{C} 12$ 

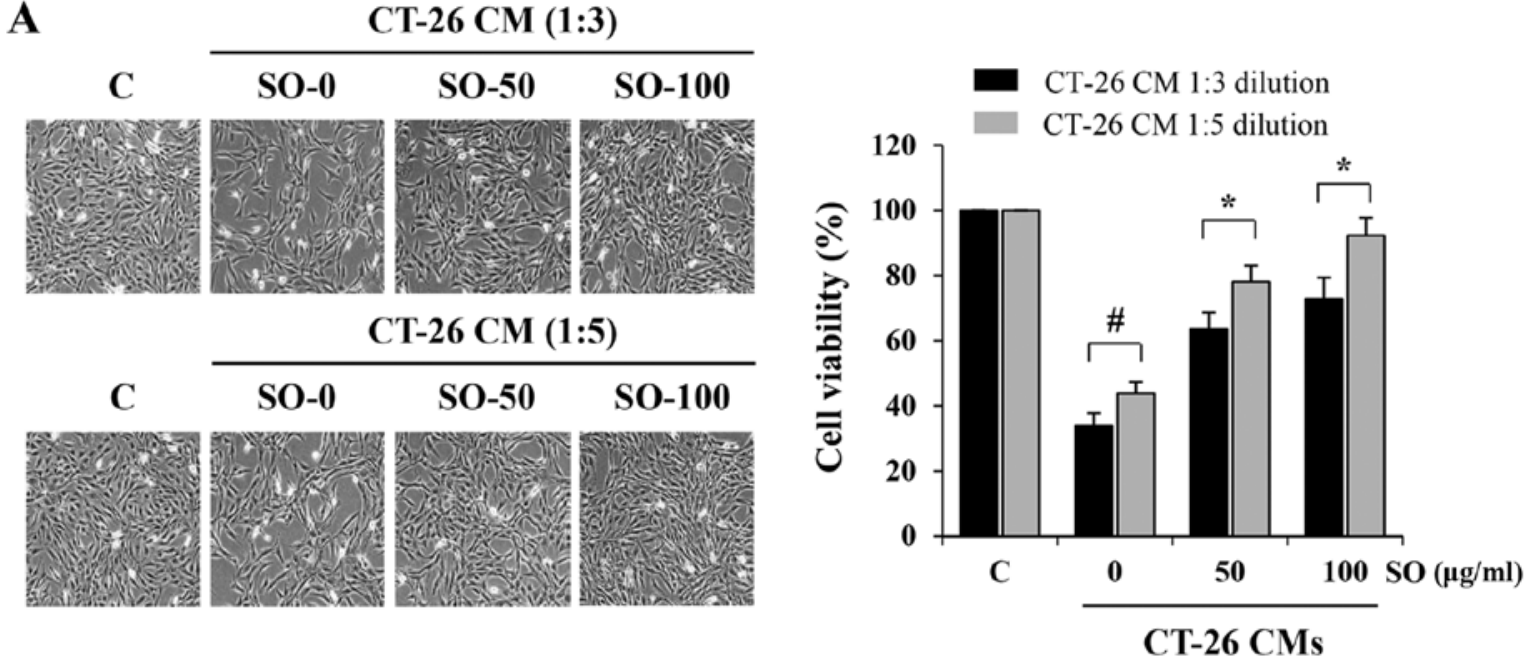

B
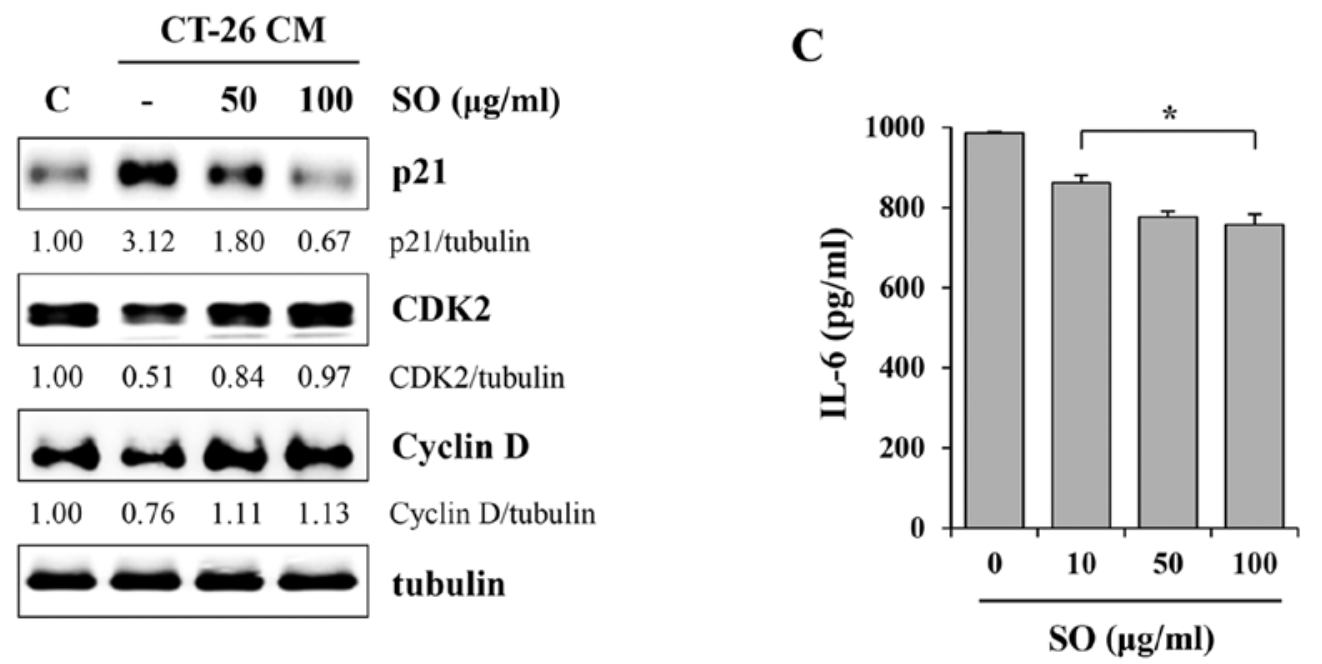

Figure 5. Effect of SO on the proliferation of C2C12 myoblasts. (A) The C2C12 myoblasts were treated with 1:3 and 1:5 dilutions of SO-treated or -untreated CT-26 conditioned medium (CM), diluted with growth medium and incubated for $36 \mathrm{~h}$. The cells were observed under an inverted microscope and viable cells were measured by the Cell Counting Kit- 8 assays. The data are means \pm SD and are representative of three independent experiments performed in triplicate. ${ }^{\#} \mathrm{P}<0.05$ vs. untreated control, $\mathrm{P}<0.05$ vs. SO-untreated control cells. (B) The expression of cell cycle-related proteins was examined by western blotting. The relative band intensities were calculated using ImageJ software after normalization using tubulin expression. (C) The levels of IL-6, IL-1 $\beta$ and TNF- $\alpha$ in the SO-treated CT-26 CMs were quantitated by ELISA. *P<0.05 vs. SO-untreated control cells.

myotube wasting, while myostatin secreted from tumors acted as a key contributor (22). To examine the effects of SO on tumor-induced muscle wasting, we evaluated $\mathrm{C} 2 \mathrm{C} 12$ myoblast proliferation after exposure to SO-treated or -untreated CT-26 $\mathrm{CM}$ at $1: 3$ or 1:5 dilutions with GM. Control CM significantly retarded $\mathrm{C} 2 \mathrm{C} 12$ myoblast growth by $\sim 65$ and $55 \%$ at $1: 3$ and $1: 5$ dilutions, respectively, compared to GM (Fig. 5A). However, compared with the control CM, SO-treated CM at $100 \mu \mathrm{g} / \mathrm{ml}$ did not cause a significant inhibition of $\mathrm{C} 2 \mathrm{C} 12$ myoblast proliferation, resulting in 28.2 and $7.7 \%$ inhibition at $1: 3$ and 1:5 dilutions, respectively, compared to GM. Since CT-26 CM inhibits myoblast proliferation by cell cycle arrest, we next examined the effect of SO-treated or -untreated CM on the expression of cell cycle-related proteins in $\mathrm{C} 2 \mathrm{C} 12$ myoblasts. Consistent with previous studies, the level of p21 was dramatically upregulated after exposure to control CM, while the levels of CDK2 and cyclin D were decreased. However, changes in these proteins in $\mathrm{C} 2 \mathrm{C} 12$ myoblasts exposed to SO-treated $\mathrm{CM}$ were insignificant, consistent with their effects on cell proliferation (Fig. 5B). Notably, the levels of IL-6 in CT-26 CM were significantly decreased by SO treatment, in a dose-dependent manner (Fig. 5C). We next examined whether SO attenuated the CT-26-mediated inhibition of $\mathrm{C} 2 \mathrm{C} 12$ myoblast differentiation. Fig. 6A shows that $\mathrm{C} 2 \mathrm{C} 12$ myoblasts differentiating in control CM (diluted to a ratio of 1:5 with DM) exhibited reduced myotube numbers as compared with the untreated control, whereas SO-treated CM slightly attenuated the impairment of $\mathrm{C} 2 \mathrm{C} 12$ myoblast differentiation. The immunoblot analysis revealed that Myh expression in myoblasts differentiating in control CM was markedly decreased compared with DM, while SO significantly prevented a CT-26-mediated reduction in Myh expression (Fig. 6B). To examine the effect of SO on $\mathrm{C} 2 \mathrm{C} 12$ myotube wasting, differentiated $\mathrm{C} 2 \mathrm{C} 12$ myotubes were incubated in SO-treated or -untreated CT-26 CM at 1:5 dilutions with DM for $48 \mathrm{~h}$. The myotubes incubated with control CM exhibited a considerable muscle wasting appearance, whereas myotubes incubated with SO-treated CM did not exhibit muscle wasting, maintaining almost intact myotubes in a similar manner to the DM-treated controls (Fig. 6C). The Myh expression in myotubes was also reduced by control CM, 
A

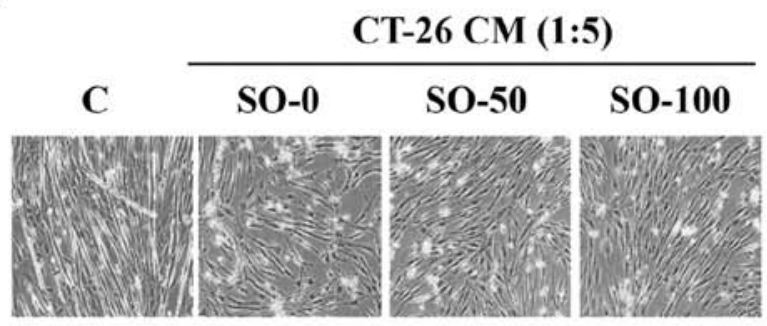

C

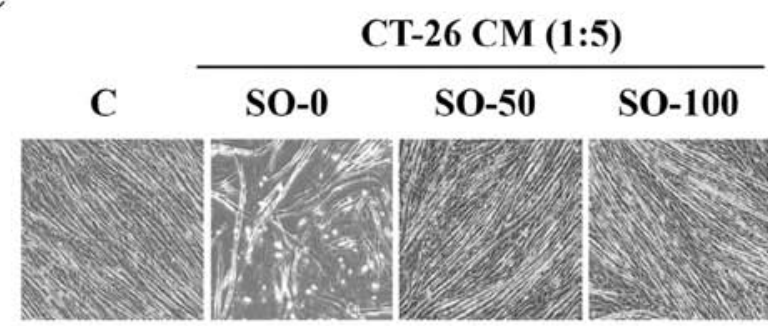

B

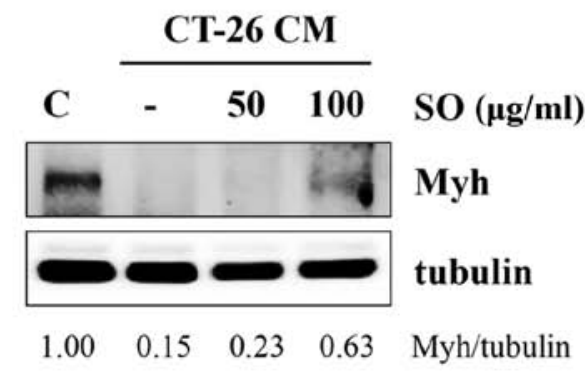

D

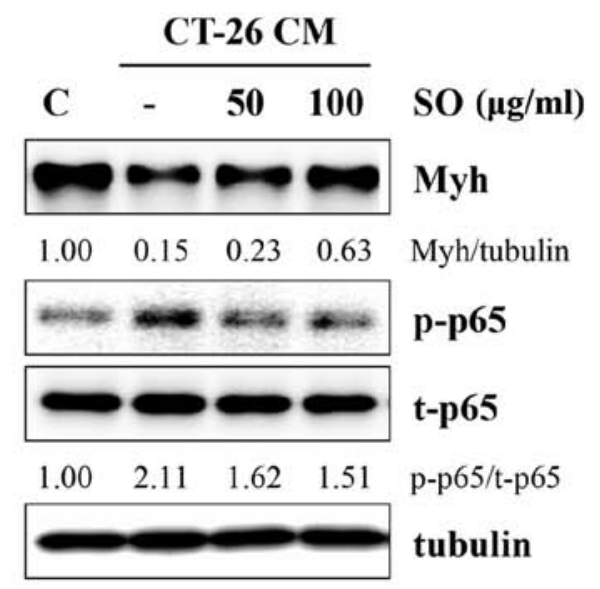

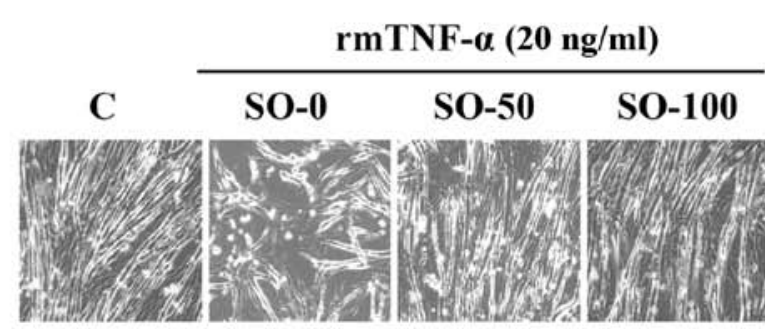

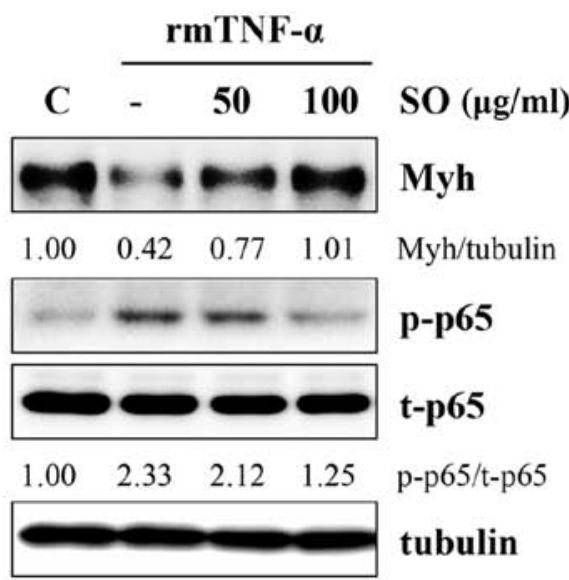

Figure 6. The effect of SO on the myogenic differentiation of $\mathrm{C} 2 \mathrm{C} 12$ myoblasts and on the wasting of $\mathrm{C} 2 \mathrm{C} 12$ myotubes. (A and B) The C2C12 myoblasts were incubated in differentiation medium (DM) or in SO-treated or -untreated CT-26 CM diluted 1:5 with DM. The content of horse serum and antibiotics was corrected to the control DM. After 4 days, cells were observed under an inverted microscope, and myosin heavy chain (Myh) expression was measured by western blotting. (C and D) The C2C12 myotubes differentiated in DMs for 4 days were incubated in DM or in the SO-treated or -untreated CT-26 CM diluted 1:5 with DM. After 48 h, myotube degradation was observed under an inverted microscope, and Myh expression was measured by western blotting. Wasting of $\mathrm{C} 2 \mathrm{C} 12$ myotubes was also examined after treatment with TNF- $\alpha(20 \mathrm{ng} / \mathrm{ml})$ with or without the indicated concentrations of SO.

while it was significantly conserved in myotubes incubated with SO-treated CM compared with DM-treated controls. In addition, control CM increased p65 phosphorylation in myotubes, while SO-treated CM did not (Fig. 6D). Previous studies reported that skeletal muscle atrophy was induced by TNF- $\alpha$, as shown by a significant decrease in cell surface myotubes derived from C2C12 cells and Myh expression (25). We confirmed that TNF- $\alpha$ induced myotube wasting and SO significantly attenuated TNF- $\alpha$-mediated skeletal muscle atrophy in $\mathrm{C} 2 \mathrm{C} 12$ cells (Fig. 6C and D).

\section{Discussion}

Cachexia is a complex metabolic syndrome characterized by anorexia, loss of skeletal muscle mass and adipose tissue, significant reduction in body weight and asthenia. It occurs in many chronic diseases, including cancer, AIDS, renal failure, diabetes and chronic obstructive pulmonary disease. Half of all cancer patients, and $80-90 \%$ of patients with tumors of pancreatic and gastric origin, exhibit a cachexia syndrome, which has a profound impact on quality of life, and causes more severe chemotherapy-related adverse events and a decreased lifespan. Most importantly, the majority of terminal cancer patients suffer from cachexia, and $22 \%$ of them die as a result of this disorder $(1-3,26)$.

Numerous cytokines secreted from the tumor or the host inflammatory cells, including TNF- $\alpha$, IL-1, IL- 6 and IFN- $\gamma$, have been postulated to act as procachectic factors that mimic leptin signaling and suppress ghrelin signaling, leading to sustained anorexia. Furthermore, these cytokines are also involved in the induction of cancer-mediated skeletal muscle wasting by inhibition of protein synthesis and/or acceleration of protein degradation. Skeletal muscle depletion is closely associated with worse outcomes in patients undergoing 
surgery, chemotherapy, and radiotherapy; therefore, reversal of skeletal muscle mass and body weight loss by inhibiting procachectic cytokines may be useful for the management of cancer patients and for an overall increase of well-being (3,6,18-20).

The current management strategies for cancer cachexia using appetite stimulants, progestational agents and orexigenic agents are limited due to their poor in vivo efficacy and high toxicity. Instead, herbal medicines have received increasing attention for use as adjuvants to enhance the efficacy and diminish complications during chemotherapy or radiation therapy. Recent studies have shown that administration of Rikkunshito, a Japanese Kampo medicine composed of eight medicinal plants, attenuated anorexia-cachexia, prolonged survival and promoted anticancer efficacy by potentiating ghrelin signaling in tumor-bearing mice $(27,28)$. Hochuekkito, a Kampo formula comprised of 10 medicinal plants, significantly attenuated marked reductions in carcass weight, food and water intake, and weight of gastrocnemius muscle and epididymal fat tissue caused by the CT-26 adenocarcinoma, by suppressing IL-6 production in macrophages (29). In addition, Sipjeondaebo-Tang (Shi-Quan-Da-Bu-Tang in Chinese and Juzen-Taiho-To in Japanese), composed of 10 species of herbs, showed therapeutic effects on cancer anorexia and cachexia by modulating body and muscle weight, food intake and cytokine and hormone production (30). Some natural herbs and their components, including Rhizoma coptidis, berberine and quercetin showed anti-cachectic effects by inhibiting tumor growth and suppressing inflammation $(31,32)$.

SO is a traditional Oriental herbal prescription that has long been used to cure alternating chills and fever from half-exterior, half-interior lesser yang disease, and has been used to treat patients with liver diseases, including hepatic fibrosis, chronic hepatitis and hepatocellular carcinoma (14-16). SO is currently prescribed for the management of fever and sore throat. Previous studies demonstrated that SO prevents depressive-like behavior in rodents by elevating serotonin and 5-hydroxyindoleacetic acid levels in the prefrontal cortex and hippocampus, inhibits thrombus formation by anti-platelet activity, and suppresses anaphylactic reaction in mast cells (33-35). Recently, we demonstrated that SO has anti-inflammatory activity in LPS-stimulated RAW 264.7 cells, by suppression of $\mathrm{NF}-\kappa \mathrm{B}$ activation and MAPK phosphorylation (17).

The present study examined whether SO administration exerted inhibitory effects on the induction of cachexia in CT-26 adenocarcinoma-bearing mice, and then determined the mechanism of action for this anti-cachectic activity. Our data showed that SO oral administration significantly inhibited tumor growth and recovered body weight compared with saline-treated control mice. SO prevented loss of skeletal muscle/fat tissue and increase in serum IL- 6 levels caused by the CT-26 tumor. We also observed that SO suppressed the production of procachectic inflammatory cytokines, including IL-6, IL-1 and TNF- $\alpha$, in macrophages through inhibition of $\mathrm{NO}$ generation and suppression of $\mathrm{p} 38, \mathrm{NF}-\kappa \mathrm{B}$ and STAT3 activation. In addition, SO prevented CT-26-mediated muscle atrophy involving proliferation, differentiation and wasting in murine $\mathrm{C} 2 \mathrm{C} 12$ myoblasts and myotubes.

In summary, the present results demonstrate that SO reduces tumor burden and systemic inflammatory responses, followed by prevention of muscle and fat degradation, indicating that $\mathrm{SO}$ is a safe and effective herbal medicine for treating cancer patients with cachexia by protecting against loss of skeletal muscle mass during catabolic conditions.

\section{Acknowledgements}

The present study was supported by Grant K15280 awarded to the Korea Institute of Oriental Medicine (KIOM) from the Ministry of Science, ICT and Future Planning (MSIP), Republic of Korea

\section{References}

1. Fearon KC, Voss AC and Hustead DS; Cancer Cachexia Study Group: Definition of cancer cachexia: Effect of weight loss, reduced food intake, and systemic inflammation on functional status and prognosis. Am J Clin Nutr 83: 1345-1350, 2006.

2. Tazi E and Errihani H: Treatment of cachexia in oncology. Indian J Palliat Care 16: 129-137, 2010.

3. Aoyagi T, Terracina KP, Raza A, Matsubara H and Takabe K: Cancer cachexia, mechanism and treatment. World J Gastrointest Oncol 7: 17-29, 2015.

4. Ozola Zalite I, Zykus R, Francisco Gonzalez M, Saygili F, Pukitis A, Gaujoux S, Charnley RM and Lyadov V: Influence of cachexia and sarcopenia on survival in pancreatic ductal adenocarcinoma: A systematic review. Pancreatology 15: 19-24, 2015.

5. Grabiec K, Burchert M, Milewska M, Błaszczyk M and Grzelkowska-Kowalczyk K: Systemic and local mechanisms leading to cachexia in cancer. Postepy Hig Med Dosw (Online) 67: 1397-1409, 2013 (In Polish).

6. Onesti JK and Guttridge DC: Inflammation based regulation of cancer cachexia. BioMed Res Int 2014: 168407, 2014.

7. Tamura S, Ouchi KF, Mori K, Endo M, Matsumoto T, Eda H, Tanaka Y, Ishitsuka H, Tokita $\mathrm{H}$ and Yamaguchi K: Involvement of human interleukin 6 in experimental cachexia induced by a human uterine cervical carcinoma xenograft. Clin Cancer Res 1: 1353-1358, 1995

8. Enomoto A, Rho MC, Fukami A, Hiraku O, Komiyama K and Hayashi M: Suppression of cancer cachexia by 20S,21-epoxy-resi bufogenin-3-acetate-a novel nonpeptide IL-6 receptor antagonist. Biochem Biophys Res Commun 323: 1096-1102, 2004.

9. Ando K, Takahashi F, Kato M, Kaneko N, Doi T, Ohe Y, Koizumi F, Nishio K and Takahashi K: Tocilizumab, a proposed therapy for the cachexia of Interleukin6-expressing lung cancer. PLoS One 9: e102436, 2014.

10. Madeddu C, Macciò A, Panzone F, Tanca FM and Mantovani G: Medroxyprogesterone acetate in the management of cancer cachexia. Expert Opin Pharmacother 10: 1359-1366, 2009.

11. Mantovani G, Macciò A, Lai P, Massa E, Ghiani M and Santona MC: Cytokine involvement in cancer anorexia/cachexia: Role of megestrol acetate and medroxyprogesterone acetate on cytokine downregulation and improvement of clinical symptoms. Crit Rev Oncog 9: 99-106, 1998.

12. Inui A: Cancer anorexia-cachexia syndrome: Current issues in research and management. CA Cancer J Clin 52: 72-91, 2002.

13. Lee JK, Kim JH and Shin HK: Therapeutic effects of the oriental herbal medicine Sho-saiko-to on liver cirrhosis and carcinoma. Hepatol Res 41: 825-837, 2011.

14. Shimizu I: Sho-saiko-to: Japanese herbal medicine for protection against hepatic fibrosis and carcinoma. J Gastroenterol Hepatol 15: D84-D90, 2000.

15. Mizushima Y, Kashii T, Tokimitsu Y and Kobayashi M: Cytotoxic effect of herbal medicine Sho-saiko-to on human lung-cancer cell-lines in vitro. Oncol Rep 2: 91-94, 1995.

16. Yano H, Mizoguchi A, Fukuda K, Haramaki M, Ogasawara S, Momosaki S and Kojiro M: The herbal medicine Sho-saiko-to inhibits proliferation of cancer cell lines by inducing apoptosis and arrest at the G0/G1 phase. Cancer Res 54: 448-454, 1994

17. Oh YC, Cho WK, Jeong YH, Im GY, Lee KJ, Yang HJ and Ma JY: Anti-inflammatory effect of Sosihotang via inhibition of nuclear factor- $\mathrm{kB}$ and mitogen-activated protein kinases signaling pathways in lipopolysaccharide-stimulated RAW 264.7 macrophage cells. Food Chem Toxicol 53: 343-351, 2013. 
18. Barton BE: IL-6-like cytokines and cancer cachexia: Consequences of chronic inflammation. Immunol Res 23: 41-58, 2001.

19. MacDonald N: Cancer cachexia and targeting chronic inflammation: A unified approach to cancer treatment and palliative/supportive care. J Support Oncol 5: 157-162, 2007.

20. Deans $C$ and Wigmore SJ: Systemic inflammation, cachexia and prognosis in patients with cancer. Curr Opin Clin Nutr Metab Care 8: 265-269, 2005.

21. Bonetto A, Aydogdu T, Kunzevitzky N, Guttridge DC, Khuri S, Koniaris LG and Zimmers TA: STAT3 activation in skeletal muscle links muscle wasting and the acute phase response in cancer cachexia. PLoS One 6: e22538, 2011.

22. Lokireddy S, Wijesoma IW, Bonala S, Wei M, Sze SK, McFarlane C, Kambadur R and Sharma M: Myostatin is a novel tumoral factor that induces cancer cachexia. Biochem J 446 : 23-36, 2012.

23. Costelli P, Muscaritoli M, Bonetto A, Penna F, Reffo P, Bossola M, Bonelli G, Doglietto GB, Baccino FM and Rossi Fanelli F: Muscle myostatin signalling is enhanced in experimental cancer cachexia. Eur J Clin Invest 38: 531-538, 2008.

24. Jespersen J, Kjaer M and Schjerling P: The possible role of myostatin in skeletal muscle atrophy and cachexia. Scand J Med Sci Sports 16: 74-82, 2006.

25. Langen RC, Schols AM, Kelders MC, Wouters EF and Janssen-Heininger YM: Inflammatory cytokines inhibit myogenic differentiation through activation of nuclear factor-kappaB. FASEB J 15: 1169-1180, 2001.

26. Donohoe CL, Ryan AM and Reynolds JV: Cancer cachexia: Mechanisms and clinical implications. Gastroenterol Res Pract 2011: 601434, 2011.

27. Fujitsuka N, Asakawa A, Amitani H, Hattori T and Inui A: Efficacy of ghrelin in cancer cachexia: Clinical trials and a novel treatment by Rikkunshito. Crit Rev Oncog 17: 277-284, 2012.
28. Fujitsuka N and Uezono Y: Rikkunshito, a ghrelin potentiator, ameliorates anorexia-cachexia syndrome. Front Pharmacol 5: 271, 2014.

29. Yae S, Takahashi F, Yae T, Yamaguchi T, Tsukada R, Koike K, Minakata K, Murakami A, Nurwidya F, Kato M, et al: Hochuekkito (TJ-41), a kampo formula, ameliorates cachexia induced by colon 26 adenocarcinoma in mice. Evid Based Complement Alternat Med 2012: 976926, 2012.

30. Choi YK, Jung KY, Woo SM, Yun YJ, Jun CY, Park JH, Shin YC, Cho SG and Ko SG: Effect of Sipjeondaebo-tang on cancer-induced anorexia and cachexia in CT-26 tumor-bearing mice. Mediators Inflamm 2014: 736563, 2014.

31. Iizuka N, Miyamoto K, Hazama S, Yoshino S, Yoshimura K, Okita K, Fukumoto T, Yamamoto S, Tangoku A and Oka M: Anticachectic effects of Coptidis rhizoma, an anti-inflammatory herb, on esophageal cancer cells that produce interleukin 6 . Cancer Lett 158: 35-41, 2000.

32. Camargo CA, da Silva ME, da Silva RA, Justo GZ, Gomes-Marcondes MC and Aoyama H: Inhibition of tumor growth by quercetin with increase of survival and prevention of cachexia in Walker 256 tumor-bearing rats. Biochem Biophys Res Commun 406: 638-642, 2011.

33. Kim HM, Kim YY, Moon HS, Lee EH, Moon SJ and An NH Inhibitory effect of anaphylactic reaction of Sosiho-Tang. Immunopharmacol Immunotoxicol 20: 567-578, 1998.

34. Lee JJ, Kim T, Cho WK and Ma JY: Antithrombotic and antiplatelet activities of Soshiho-tang extract. BMC Complement Altern Med 13: 137, 2013

35. Su GY, Yang JY, Wang F, Xiong ZL, Hou Y, Zhang K, Song C, Ma J, Song SJ, Teng HF, et al: Xiaochaihutang prevents depressive-like behaviour in rodents by enhancing the serotonergic system. J Pharm Pharmacol 66: 823-834, 2014. 